\title{
The Influence of the Sample Shape on the Flux Jumps Dynamics in Conventional NbTi Superconductor
}

\author{
A. NabialeK ${ }^{a, *}, \mathrm{~S} \mathrm{VAsiliev}^{b}, \mathrm{~V} \cdot \mathrm{Chabanenko}^{b}, \mathrm{~V} \cdot \mathrm{Rusakov}^{c}$, \\ S. PIECHOTA ${ }^{a}$ AND H. SZYMCZAK ${ }^{a}$ \\ ${ }^{a}$ Institute of Physics, Polish Academy of Sciences \\ al. Lotników 32/46, 02-668 Warsaw, Poland \\ ${ }^{b}$ Donetsk Physico-Technical Institute, Ukrainian Academy of Sciences \\ 72 R. Luxemburg str., 83114, Donetsk, Ukraine
}

${ }^{c}$ Donetsk National University, 24 Universitetskaya str., 83055, Donetsk, Ukraine

We investigated giant flux jumps, caused by thermomagnetic avalanches, in two cylindrical samples of conventional NbTi-50\% superconductor of the same diameter $-14 \mathrm{~mm}$, but of different heights $-4 \mathrm{~mm}$ and $20 \mathrm{~mm}$. We studied the influence of demagnetizing effects on the flux jumps occurrence, the amount of the magnetic flux entering the sample during the following jumps and on the flux jumps dynamics. The dynamics of the magnetic flux entering the sample is compared with the dynamics of the stray field.

PACS numbers: 74.70.Ad, 74.25.Ha

\section{Introduction}

Giant flux jumps caused by thermomagnetic avalanches are commonly observed in hard type-II superconductors [1]. This phenomenon is problematic from the viewpoint of applications of the superconductors, because the thermomagnetic avalanche may drive the superconducting sample into a normal state. Studies of the avalanche-like processes, which are strongly nonlinear, are also interesting from theoretical point of view. The dynamics of the thermomagnetic avalanche remains poorly understood and needs further investigations [2]. It is known that demagnetizing effects strongly influence conditions of the flux jumps occurrence [3]. In thin films, at certain conditions, the avalanche of magnetic flux may propagate in the form of dendrites [4]. Recently, this phenomenon was intensively studied both experimentally and theoretically [5]. In our previous work [6] we studied the

*corresponding author; e-mail: nabia@ifpan.edu.pl 
structure of the thermomagnetic avalanches in superconducting NbTi disk. The aim of the present work is to compare the structure of thermomagnetic avalanches in two bulk samples of conventional superconductor of different demagnetizing factors. In particular, we study the range of the flux jumps occurrence, the amount of the magnetic flux entering the sample during the following flux jumps and the dynamics of the magnetic flux entering the sample as well as the dynamics of the stray field.

\section{Experiment}

Both samples, studied in our experiment, were cut from the same long cylinder of NbTi-50\% superconductor with the critical temperature of $9.6 \mathrm{~K}$ and characterized by the critical current density of about $10^{9} \mathrm{~A} / \mathrm{m}^{2}$, at $4.2 \mathrm{~K}$ in zero external magnetic field. They have the same diameter of $14 \mathrm{~mm}$ and heights of $4 \mathrm{~mm}$ (disk) and $20 \mathrm{~mm}$ (cylinder), respectively.

Flux dynamics inside superconductors was investigated by pick-up coils. These coils were wound directly on the superconducting samples, in the middle of their heights, and consisted of 10 turns of copper wire. We also investigated the changes of the magnetic flux around the samples. To this aim, we used outside pick-up coils, whose cross-section embraced some area around the samples, and consisted of 5 turns of copper wire (see Ref. [6] for details). These coils detected the changes of the stray field. The thermomagnetic avalanches induced a voltage proportional to the time derivative of the magnetic flux in pick-up coils. This voltage was registered by a transient recorder with memory. The measurements of the local surface induction were performed by using a miniature Hall probe. Our investigations were performed in a $12 \mathrm{~T}$ superconducting magnet with a variable temperature insert. The external magnetic field was parallel to the axes of the investigated samples with cylindrical symmetry.

\section{Results}

Figure 1 presents the surface magnetization hysteresis loop, $M_{\text {surf }}\left(H_{\text {ext }}\right)=$ $B_{\text {surf }}\left(H_{\text {ext }}\right)-\mu_{0} H_{\text {ext }}$, taken for the disk and for the cylinder at $4.2 \mathrm{~K}$. The Hall probe, which measured the surface induction $B_{\text {surf }}\left(H_{\text {ext }}\right)$, was placed each time in the middle of the sample surface. It is clearly seen that the range of the flux jumps occurrence is more restricted in the case of the disk $( \pm 2 \mathrm{~T})$ than in the case of the cylinder $( \pm 5 \mathrm{~T})$. In the case of the cylinder some additional jumps also occur in the external magnetic field higher than $9 \mathrm{~T}$.

After integration in time signals taken from the pick-up coil wound directly on the sample, we obtained the amount of the magnetic flux entering the sample during the following flux jumps. The comparison of the amount of magnetic flux entering the disk and the cylinder, studied in our experiment at the temperature of $4.2 \mathrm{~K}$ during the following jump, is schematically shown in Fig. 2. Data points shown in Fig. 2 correspond to the following jumps, shown in Fig. 1. One can see 




Fig. 1. Surface magnetization hysteresis loops for a disk - upper part, and a cylinder - lower part, of a NbTi-50\% superconductor, taken at $4.2 \mathrm{~K}$.



Fig. 2. The amount of the magnetic flux entering the sample during the following flux jumps, for the disk and the cylinder.

that the amount of the flux entering the sample is approximately twice smaller in the case of the disk than in the case of the cylinder. Additionally, this amount of magnetic flux, statistically, decreases with an increase in the external magnetic field.

Duration of the following jumps, registered by the pick-up coil wound directly on the sample, for both samples studied in our experiment, is shown in Fig. 3. One can see that this duration is shorter in the case of the disk. 




Fig. 3. Duration of the following flux jumps, for the disk and the cylinder. The dynamics of the jumps was registered by the pick-up coil wound directly on the sample.

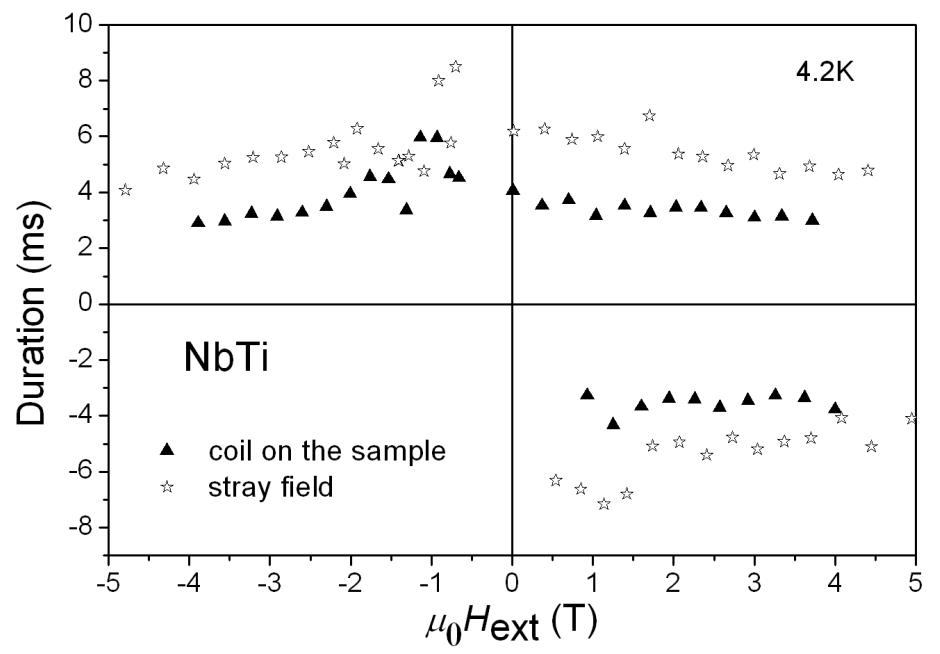

Fig. 4. Comparison of the duration of the flux jumps registered by the pick-up coil wound directly on the sample and by the pick-up coil that measured the changes of the stray field around the NbTi cylinder.

Figure 4 presents the comparison of the duration of the jumps registered by the pick-up coil wound directly on the sample and by the pick-up coil that measured the changes of the stray field around the NbTi cylinder. The duration of the stray field jumps is significantly longer than duration of the magnetic flux entrance into the investigated cylinder. 


\section{Model}

In order to analyze, quantitatively, the influence of the sample geometry on the amount of the magnetic flux entering the sample during flux jumps, we have developed the following model. We considered an infinitely long rod with rectangular cross-section, with diameter $2 a$ and height $h$ (see Fig. 5). We assume the

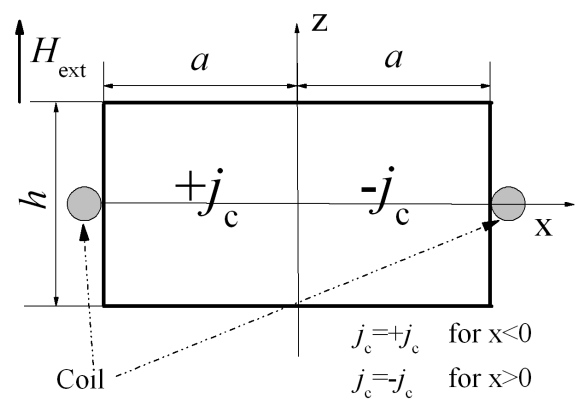

Fig. 5. The cross-section of the sample considered in our model.

sample to be fully penetrated by the magnetic flux and the absolute value of the critical current density, $j_{\mathrm{c}}$, to be constant in the whole volume of the superconducting sample. In this case, it is possible to obtain analytical formula for the distribution of the $z$-component of the magnetic field in the plane $z=0$. After integration of the magnetic field distribution over the sample diameter, we obtain the formula for the magnetic flux, $\Phi$, whose changes are measured by the coil in our experiment

$$
\Phi\left(j_{\mathrm{c}}, a, \xi, k\right)=\mu_{0} j_{\mathrm{c}} a^{2} \xi F(k),
$$

where $\xi$ is the length of the slab and $k=a / h$. The function $F(k)$ is given by the following analytical formula:

$$
\begin{aligned}
F(k) & =\left(-\frac{1}{2 \pi}\right)\left[\pi\left(1-\frac{1}{2 k^{2}}\right)+\frac{2}{k^{2}} \arctan (2 k)\right. \\
& -2\left(1+\frac{1}{4 k^{2}}\right) \arctan (4 k)-10 \arctan \left(\frac{1}{4 k}\right) \\
& \left.+4\left(1+\frac{1}{4 k^{2}}\right) \arctan \left(\frac{1}{2 k}\right)+\frac{2}{k} \ln \left(\frac{1+4 k^{2}}{1+16 k^{2}}\right)\right] .
\end{aligned}
$$

In the case of infinite slab sample $(h \rightarrow \infty$ and $k \rightarrow 0), F(k \rightarrow 0) \rightarrow 1$. With a decrease in the sample height, it means with increasing $k$, the function $F(k)$ decreases, approaching zero for $k \rightarrow \infty$.

\section{Discussion}

Our experimental results show (Fig. 1) that with a decrease in sample height the range of the flux jumps occurrence becomes more restricted. This fact can be understood taking into account a decrease of the field in full penetration $[7,8]$. Flux 
jumps occur when the difference between the magnetic field in two points of the superconducting sample (usually between the magnetic field in the sample center and the magnetic field at the sample edge) reaches some critical value [1]. If the sample is fully penetrated, and assuming that the critical current density decreases with increasing magnetic field, this difference cannot increase any more and flux jumps disappear. In increasing external magnetic field, the field of full penetration is reached earlier in the case of the disk than in the case of the cylinder. Hence, the range of the flux jumps is more restricted in the case of the disk. Flux jumps that occur in the cylinder in the external magnetic field higher than about $9 \mathrm{~T}$ are similar to the so-called "island jumps", which may occur when the critical current density increases, in some range, with increasing external magnetic field. The conditions of the occurrence of the "island jumps" were analyzed in our previous work [9].

According to our simple model one can expect a decrease in the amount of the magnetic flux entering the sample with a decrease in the sample height. Such dependence was indeed observed in our experiment (see Fig. 2). Taking the values of a diameter and heights of our samples, we obtain $k_{\text {disk }}=1.75$ and $k_{\text {cylinder }}=0.35$. Hence (see Eq. $\left.(2)\right), F\left(k_{\text {cylinder }}\right) / F\left(k_{\text {disk }}\right) \approx 2.9$, which is slightly higher than the ratio of the amounts of the magnetic flux entering the cylinder and the disk, studied in our experiment (see Fig. 2). This small discrepancy can be understood bearing in mind rather rough assumptions of our model. As the magnetic flux, $\Phi$, is proportional to the critical current density (see Eq. (1)), we can also understand a decrease in the magnetic flux entering the sample with increasing external magnetic field (see Fig. 2).

As the field of full penetration is smaller in the case of the disk [8], the magnetic field penetrates this sample more rapidly, and the time of the following flux jumps is shorter than in the case of the cylinder (see Fig. 3).

Similarly to the case of the disk [6], in the case of the cylinder the duration of the stray field jump is significantly longer than the duration of the magnetic flux entrance into the volume of the investigated sample (see Fig. 4). This experimental fact suggests that when the process of the magnetic flux entrance (registered by the coil wound directly on the sample) is finished, the process of the magnetic flux redistribution in the superconductor's volume (which manifests itself by changes of the stray field) is still continuing. These two processes are even more distinguishable in the case of the cylinder than in the case of the disk, because in the case of the cylinder they occur in a larger volume.

\section{Conclusions}

With an increase in the sample height: (1) the range of the flux jumps occurrence becomes wider, (2) the amount of the magnetic flux entering the sample during the following jumps increases, (3) the duration of the following jumps increases, (4) the process of the magnetic flux redistribution becomes longer. These 
experimental facts can be qualitatively understood bearing in mind changes of the field of full penetration as well as a simple theoretical model, which correlates the amount of the magnetic flux with sample geometry.

\section{Acknowledgments}

This work was partly supported by Polish Ministry of Science and Higher Education under research project for years 2007-2009 (grant N N202 1285 33).

\section{References}

[1] R.G. Mints, A.L. Rakhmanov, Rev. Mod. Phys. 53, 551 (1981).

[2] A. Nabiałek, S. Vasiliev, P. Aleksyeyev, V. Chabanenko, Acta Phys. Pol. A 111, 153 (2007).

[3] A. Nabiałek, M. Niewczas, Physica C 436, 43 (2006).

[4] T.J. Johansen, M. Baziljevich, D.V. Shantsev, P.E. Goa, Y.M. Galperin, W.N. Kang, H.J. Kim, E.M. Choi, M.S. Kim, S.I. Lee, Europhys. Lett. 59, 559 (2002).

[5] D.V. Denisov, A.L. Rakhmanov, D.V. Shantsev, Y.M. Galperin, T.H. Johansen, Phys. Rev. B 73, 014512 (2006).

[6] S. Vasiliev, A. Nabiałek, V. Chabanenko, V. Rusakov, S. Piechota, H. Szymczak, Acta Phys. Pol. A 109, 661 (2006).

[7] E.H. Brandt, Phys. Rev. B 54, 4246 (1996).

[8] E.H. Brandt, Phys. Rev. B 58, 6506 (1998).

[9] V.V. Chabanenko, V.F. Rusakov, A.I. D'yachenko, S. Piechota, A. Nabialek, H. Szymczak, Physica C 341-348, 2031 (2000). 